\title{
Discrete Power Allocation via Ant Colony Optimization for Multi-cell OFDM Systems
}

\author{
Da Wang * , Xiaodong Xu *, Xin Chen *, Xiaofeng Tao *, Yue Yin *, Harald Haas ${ }^{\dagger}$ \\ * Wireless Technology Innovation Institute, Key Laboratory of Universal Wireless Communication, Ministry of Education \\ Beijing University of Posts and Telecommunications, Beijing 100876, P. R. China. \\ $\dagger$ Institute for Digital Communications, School of Engineering and Electronics \\ The University of Edinburgh Edinburgh, EH9 3JL, UK \\ Email: wonder722@gmail.com
}

\begin{abstract}
The majority studies on resource allocation are based on continuous power allocation. However, the transmit power can be assumed as a finite set of discrete power levels only, especially for practical digital cellular systems. By simply rounding up or down, the conventional continuous power allocation algorithm will not suffice. In this paper, via transmitting signal on discrete power levels, multi-cell power allocation is modeled as a combinatorial optimization problem, which proved to be NP-hard. Ant colony optimization (ACO) is applied to get nearoptimal solution of the problem. In particular, a multiple power level based searching graph is designed, and conventional ACO is improved that ant colonies in each cell cooperate to maximize system throughput. Simulation results indicate that with only 4 power levels, the proposed algorithm can achieve a significant rate gain. And the throughput improvement increases to roughly $12 \%$ and $33 \%$ compared with binary power allocation and equal power allocation
\end{abstract}

\section{INTRODUCTION}

Due to its potential in supporting high data rate services over frequency selective fading channels, orthogonal frequency division multiplexing (OFDM) based multiple access has been accepted as the downlink access technology of 3rd generation partnership project (3GPP) long term evolution (LTE) and LTE-Advanced standard [1]. However, cochannel interference from neighboring cells degrades the performance of multicell OFDM systems significantly. Therefore, it is important to develop a good radio resource allocation scheme which reduces inter-cell interference (ICI) and improves the system performance effectively.

An improved iterative water-filling resource allocation algorithm for multi-cell scenarios is proposed in [2], which maximizes the weighted system sum-rate while subject to the power constraints of each base station. In [3], multi-cell resource allocation is modeled as a noncooperative power allocation game (NPAG), and an iterative algorithm is proposed to reach Nash equilibrium (NE). In [4], a resource allocation algorithm combined with interference coordination is proposed. However, most of the conventional algorithms, like the previous mentioned, are proposed based on continuous power allocation. Actually, the transmit power can be assumed as a finite set of discrete power levels only, especially for practical digital cellular systems. And it has been proved in [5] that by simply rounding up or down, the convergence and uniqueness of the continuous power algorithm are lost.
In [6], Anders Gjendemsø proposes a binary power allocation (BPA) algorithm for maximizing the throughput of multi-cell systems. BPA mitigates ICI by deciding whether the subchannel to be silent or transmitting at maximum power. It is shown that by adopting binary power levels, the search for distributed algorithms becomes more manageable and the affection on system performance is minor. In [7], Honghai Zhang maximizes the weighted sum-rate in multi-cell networks through discrete power allocation and coordinated scheduling. He also noted that, for practical systems, discrete power allocation offers two main benefits over conventional continuous power allocation: (i) the transmitter design is much simplified and more importantly (ii) the overhead of information exchange among network nodes can be reduced. However, discrete power allocation for multi-cell systems is an NP-hard combinational optimization problem [7], difficult to obtain its optimal solution in polynomial time.

Metaheuristic algorithm is an efficient method to obtain near-optimal solutions of NP-hard problems. Up to now, some works have been done on resource allocation based on metaheuristic algorithm. In [8], a Genetic Algorithm (GA) based scheme is proposed to minimize the overall transmit power with the QoS constraints of users. In [9], both GA and Particle Swarm Optimization (PSO) are applied for subcarrier allocation while the bit allocation part is based on water-filling algorithm. Ant colony optimization (ACO) is adopted to solve resource allocation problem in [10], where a multiple edges ACO graphic is developed to allocated subchannels and bit jointly. However, the schemes discussed above are all intend for single cell system, which can not be applied to multicell environment directly. A two level ACO based multi-cell subchannel allocation scheme is proposed in [11]. However, the scheme didn't consider appropriate power allocation by using an equal power allocation.

In this paper, ACO is applied to solve discrete power allocation problem for multi-cell OFDM systems. First, a multiple power level based searching graph is designed. Then, as the multi-cell discrete power allocation problem is much more complex than typical NP-hard problems, conventional ACO algorithm is improved that ant colonies in each cell cooperate with each other to maximize the system throughput. Finally, system level simulation is performed to verify the 
effectiveness and feasibility of the proposed scheme.

The remainder of this paper is organized as follows. Section II shows the system model and formulates an optimal system throughput problem. The proposed algorithm based on ACO is described in details in section III. Section IV presents the simulation results and compares the performance with the existing methods. Finally, conclusion are given in section V.

\section{System Model And Problem Formulation}

Consider a downlink multi-cell OFDM system consisting of $I(I \geq 2)$ cochannel cells, where $M$ subchannels are fully reused in the system. $K$ users are randomly distributed in each cell. Base stations and users are equipped with one receive and one transmit antenna, respectively. Assumed that each subchannel of a cell can be assigned to only one user, and the system has knowledge of the instantaneous channel state information (CSI) for all users. The continuous transmit power domain for each subchannel is quantized into $L(L \geq 2)$ discrete power levels $0=\varepsilon_{1}<\varepsilon_{2}<\cdots<\varepsilon_{L}=\varepsilon_{\max }$, where $\varepsilon_{\max }$ is the maximum transmit power of the subchannel. An increase of $L$ leads to the fact that the discrete domain is much closer to a continuous one, but meanwhile computational complexity increases as well.

Assume user $k$ is connected to the $i$ th cell on subchannel $m$. Denote $s_{m, k}^{i}$ is the complex symbol transmitted from the base station to user $k$. When the ICI from adjacent cells is considered, the received signal of user $k$ can be given by:

$$
y_{m, k}^{i}=\sqrt{P_{m}^{i}} H_{m, k}^{i} s_{m, k}^{i}+\sum_{j \neq i}^{I} \sqrt{P_{m}^{j}} H_{m, k}^{j} s_{m, k}^{j}+n_{m, k}^{i},
$$

where $P_{m}^{i}$ is the transmit power on subchannel $m$ of cell $i$, which is restrict to a discrete set of values $S=\left\{\varepsilon_{1}, \varepsilon_{2}, \cdots\right.$ $\left.\cdot, \varepsilon_{L}\right\} . H_{m, k}^{i}$ is the complex channel response between cell $i$ and user $k$ on subchannel $m . n_{m, k}^{i}$ is the additive white Gaussian noise with noise power $N_{0}$. We indicate with $\mathbf{P}_{m}=$ $\left[P_{m}^{1}, P_{m}^{2}, \cdots, P_{m}^{I}\right]$ the power strategy of subchannel $m$ of $I$ cochannel cells, the received signal to interference plus noise ratio (SINR) of user $k$ on subchannel $m$ of cell $i$ can be written as a function of $\mathbf{P}_{m}$ given by (2).

$$
\operatorname{SINR}_{m, k}^{i}\left(\mathbf{P}_{m}\right)=\frac{P_{m}^{i}\left|H_{m, k}^{i}\right|^{2}}{\sum_{j \neq i}^{I} P_{m}^{j}\left|H_{m, k}^{j}\right|^{2}+N_{0}} .
$$

According to Shannon theorem, the achievable rate on subchannel $m$ for user $k$ can be expressed as:

$$
r_{m, k}^{i}\left(\mathbf{P}_{m}\right)=\frac{W}{M} \log _{2}\left(1+\frac{\operatorname{SINR}_{m, k}^{i}\left(\mathbf{P}_{m}\right)}{\Gamma}\right),
$$

where $W$ is the system bandwidth, $\Gamma$ is the SINR gap. For multilevel quadrature amplitude modulation (MQAM), $\Gamma$ is a constant related to the required bit error rate (BER) [12]:

$$
\Gamma=-\ln \left(5 B E R_{r e q}\right) / 1.5
$$

For simplicity, we assume that all the subchannels have been assigned to certain users. Define the system throughput as the sum transmission rate of the users in the considered system. The discrete power allocation optimization problem of maximizing system throughput can be formulated as:

$$
\begin{array}{cl}
\max & R=\sum_{i=1}^{I} \sum_{m=1}^{M} r_{m, k}^{i}\left(\mathbf{P}_{m}\right) \\
\text { s.t. } & \sum_{m=1}^{M} P_{m}^{i} \leq P_{\max } \forall i, \\
& P_{m}^{i} \in\left\{\varepsilon_{1}, \varepsilon_{2}, \cdots, \varepsilon_{L}\right\}, \\
& B E R_{m, k}^{i}=B E R_{\mathrm{tar}},
\end{array}
$$

where $P_{\max }$ is maximum transmit power of the BSs. The combinatorial optimization problem (5) is an NP-hard problem [7], [13]. This is because even the sum-rate maximization problem with binary power allocation for a given user assignment is proved to be NP-hard in the proof of Theorem 1 in [13]. Exhaustive searching is a possible method to obtain its optimal solutions, but it is not able to be implemented in practical systems due to intensive computing. Therefore, ACO is used in this paper to provide solutions in real-time.

\section{ACO-BASED SOLUTION}

As one of metaheuristic algorithms, ACO is first proposed by Marco Dorigo to solve traveling salesman problem (TSP) [14], and became well-known due to is advantage in solving combinatorial optimization problems. It is inspired by the highly collaborative behavior of ants finding the shortest path between food and nest. Natural ants are abstracted as basic computational agents with simple capabilities, and their main task is to find the best complete path. Evaporable pheromone is released on the path that the artificial ant has passed, and the released amount is proportional to the quality of the solution that the path indicates, thus a positive feedback mechanism can be formed. As a result, after several times of iteration, all the ants gradually converge to one suboptimal or sometimes optimal path for the target problem.

Used in different NP-hard problems, ACO has been proved qualified with excellent robustness and easy to be applied in realistic environment. The first ACO algorithm is ant system (AS) [14], and several improved algorithm has been proposed after its inspiration. In this paper, ant colony system (ACS) [15], a more efficient ACO algorithm, is applied.

\section{A. Searching Graph Construction}

First of all, the searching graph of the discrete power allocation problem should be designed for the ants to move from node to node to construct solutions. In this paper, each cell creates a searching graph for ants to allocate power for the subchannels. Since multi-cell power allocation problem is more complex than typical NP-hard problem, like TSP, the conventional ACO is improved that ant colonies of each cell cooperate with each other to maximize the whole system throughput. The searching graph of each cell is shown in Fig. 1 (a), which is a two-dimensional graph consists of $L \times M$ nodes. The Column denotes $M$ subchannels, and 
the row denotes $L$ power levels, thus each node represents a subchannel and power level pair. Virtual nest and food are located on both sides of the graph. Artificial ants start out from the nest and move from one column to another to select power level for the subchannels until food resource is found. The size of the nodes represents for the pheromone amount, and at the beginning of the algorithm, the pheromone amount for all the nodes is initialized to an equal const value $\tau_{0}$. By continually changing the size of the nodes while ants moving, ACO is able to obtain satisfied feasible solutions. Fig. 1 (b) shows the searching graph after several times of iterations, which also give an example of one complete path: $P_{1}^{i}=\varepsilon_{3}, P_{2}^{i}=\varepsilon_{2}, P_{3}^{i}=\varepsilon_{L}, \cdots, P_{M-1}^{i}=\varepsilon_{1}, P_{M}^{i}=\varepsilon_{3}$.

\section{B. Heuristic Information Calculation}

Pheromone is not the only factor that the ants depend on to construct solutions of the target problem, heuristic information also plays an important role when the ants chose the next step. Similar to the visibility of ant in TSP, this information measures the quality of nodes that can be added to the current partial solution. The bigger the heuristic information of the node is, the more possibly to be selected. Power allocation strategies of adjacent cells influence with each other, high power level produces high data rate of the specific cell, but causes serious interference to neighboring cells. Hence, to attract the ants finding global optimal solutions, the performance of cochannel cells is also taken into consideration of the calculation of the heuristic information. We define:

$$
R_{l, m}=\sum_{j=1}^{I} r_{m, k}^{j}\left(\mathbf{P}_{m} \mid P_{m}^{i}=\varepsilon_{l}\right),
$$

where the power level of subchannel $m$ of the concerned cell $i$ is $l$, and for other cells the power level of subchannel $m$ is depend on the best-so-far solution, whose initial value is a random solution satisfied power constraint. Furthermore, $R_{l, m}$ is normalized and define the heuristic information $\eta_{l, m}$ for node $(l, m)$ as:

$$
\eta_{l, m}=\frac{R_{l, m}}{\sum_{k=1}^{L} R_{k, m}} .
$$

\section{Migration of Ants}

At each iteration, an artificial ant is dispatched from the nest to find possible solution for the cell, and the computation of each cell runs independently. The ants choose power level for the subchannel by traveling in the searching graph according to a pseudorandom proportional rule which offers a tradeoff between "exploitation" and "biased exploration" [15]. Assuming there is an ant lies on column $m$, the rule can be described as:

$l=\left\{\begin{array}{cc}\arg \max _{k \in[1,2, \cdots, L]}\left(\left[\tau_{k, m}\right]^{\alpha}\left[\eta_{k, m}\right]^{\beta}\right), & \text { if } q \leq q_{0} \\ J, & \text { else }\end{array}\right.$,

where $q$ is a random number uniformly distributed in $[0,1], q_{0}$ is a parameter satisfying $0 \leq q_{0} \leq 1, \alpha$ and $\beta$ are parameters

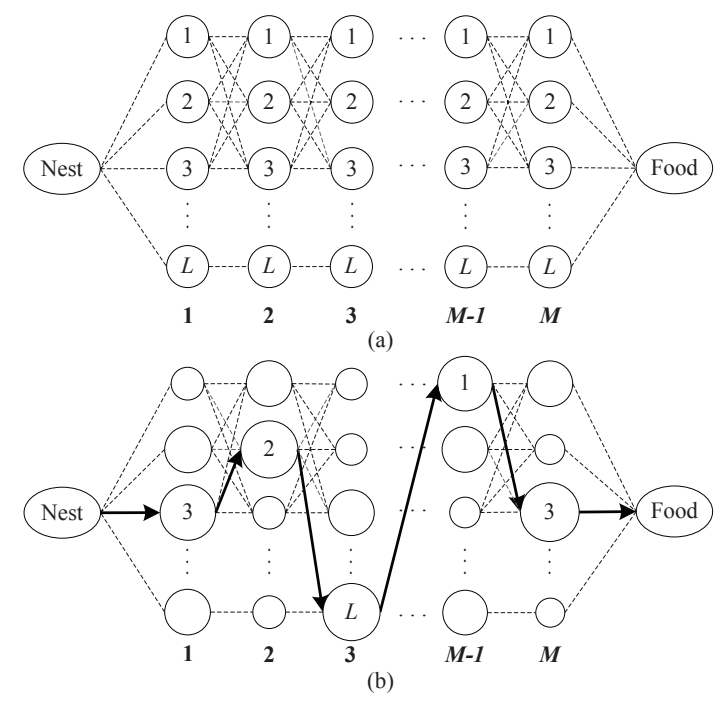

Fig. 1. (a) The searching graph of each cell at the beginning of the algorithm; (b) The searching graph of each cell after several times of iteration.

determine the relative importance between pheromone information and heuristic information. When $q \leq q_{0}$, exploitation is performed that the current best power level is selected, otherwise, biased exploration is applied and we choose the power level $J$ based on roulette wheel selection (RWS) using the following probability distribution of the candidate nodes:

$$
p(l, m)=\frac{\left[\tau_{l, m}\right]^{\alpha}\left[\eta_{l, m}\right]^{\beta}}{\sum_{k=1}^{L}\left[\tau_{k, m}\right]^{\alpha}\left[\eta_{k, m}\right]^{\beta}}, \forall l,
$$

where $p(l, m)$ represents for the possibility of node $(l, m)$ to be selected as the next step.

One thing improtant to mention, during the execution of the migration, if the power summation of the subchannels that have been allocated unexpectedly exceeds power constraint of the base station, the ant stops moving any more, and is eliminated immediately. Another ant is dispatched form the nest to replace the eliminated one and restart allocating power for all subchannels until a complete path is finished.

\section{Pheromone Updating}

The pheromone updating process includes pheromone evaporation and release. Once the ants move a step, i.e. select a power level for a subchannel, the local pheromone updating is conducted on the corresponding node based on the following formula:

$$
\tau_{l, m}=(1-\xi) \tau_{l, m}+\xi \tau_{0}
$$

where $\xi$ is the local evaporation rate. Local pheromone updating can reduce the pheromone amount and improve the possibility of other nodes to be select by the ants. In this way, the algorithm will not slide into stagnation, which means the ants will not select the same path, and this is effective for the situation when power exceeds constraint.

Global pheromone updating is based on the information exchange between ant colonies of different cells. Once all the 
ant colonies of different cells finished the power allocation for their subchannels, new power allocation strategy is gathered from different cells and compared with the best-so-far solution. The one which can provide larger system throughput will be the new best-so-far solution. And the global pheromone updating is applied only on the nodes indicated by the bestso-far solution based on the following formula:

$$
\tau_{l, m}=(1-\rho) \tau_{l, m}+k_{0} \rho R,
$$

where $\rho$ is the global evaporation rate, $k_{0}$ is a const parameter to adjust the amount of pheromone to be released. We can see from (11), the better the solution is, the more pheromone is gained on the nodes of path. Furthermore, new best-so-far solution is passed on to each colony.

After the construction of global pheromone updating, one time of iteration is finished. If the condition of iteration ends is satisfied, for instance reaching the pre-determined maximal iterative time, the algorithm will be finished and the power is allocated based on the best-so-far solution. The proposed ACO-based algorithm can be summarized in Fig. 2, where $t$ denotes the iterative times.

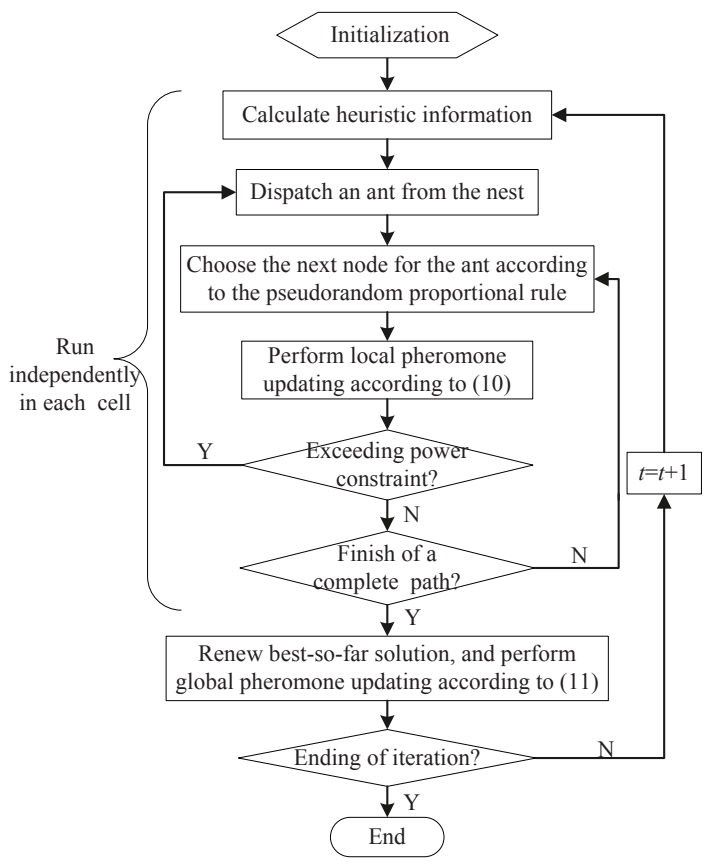

Fig. 2. Flowchart of the ACO-based algorithm.

\section{Simulation Results and Analysis}

In this section, Monte Carlo simulation have been carried out to illustrate the performance of the proposed algorithm. We set up a seven-cell simulation scenario, where six cells around a central cell with omnidirectional antennas with the same cell radius. To reduce the simulation complexiy, assume that 10 subchannels are available for each cell. A simple fair subchannel allocation scheme is deployed, thus each user is allocated same number of subchannels. More detailed simulation parameters are listed in Table I. The maximize
TABLE I

SIMULATION PARAMETERS

\begin{tabular}{c|c}
\hline \hline Parameters & Value \\
\hline Cell radius & $500 \mathrm{~m}$ \\
Subchannel number & 10 \\
Total BS TX power & $46 \mathrm{dBm}$ \\
Carrier frequency & $2 \mathrm{GHz}$ \\
Bandwidth & $10 \mathrm{MHz}$ \\
Path loss (dB) & $L=128.1+37.6 \log _{10}(R), R / \mathrm{km}$ \\
Shadowing standard deviation & $8 \mathrm{~dB}$ \\
Shadowing correlation distance & $50 \mathrm{~m}$ \\
Fast fading model & $\mathrm{SCME}$ \\
\hline \hline
\end{tabular}

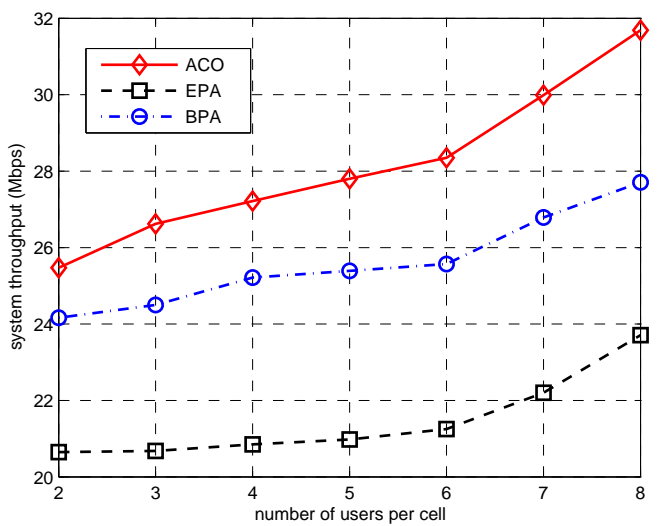

Fig. 3. System throughput of three different schemes with $L=4$.

iterative times is set $T=100$. For the ACO parameters, it is found the following parameters producing good performance in a series of problems [16]: $\tau_{0}=0.5, \alpha=1, \beta=3, q_{0}=0.9$, $\xi=\rho=0.1$.

In order to make comparison with existing algorithms, we also give out the simulation results of BPA given by greedy approach in [6], and equal power allocation (EPA) which the transmit power on every subchannel is equal.

In Fig. 3, the throughput of three schemes is shown as a function of the number of users in each cell. For ACO, the power level number is 4 . We can come to the conclusion that ACO achieves the best performance, and the throughput improvement increases to roughly $12 \%$ and $33 \%$ compared with BPA and EPA. Due to the limitation of binary power levels and greedy approach, which just chooses current best solution, the throughput of BPA is lower than ACO. In addition, the performance gap between BPA and ACO becomes large as the number of users increases, which can be concluded that the proposed algorithm employs multi-user and multi-cell diversity well.

In Fig. 4 (a), we compare the performance of ACO for different number of power levels. It can be concluded that increasing power level number from 2 to 4 gives noticeable system throughput gain, but further increasing the number of transmit power levels beyond 4 only gives limited performance 

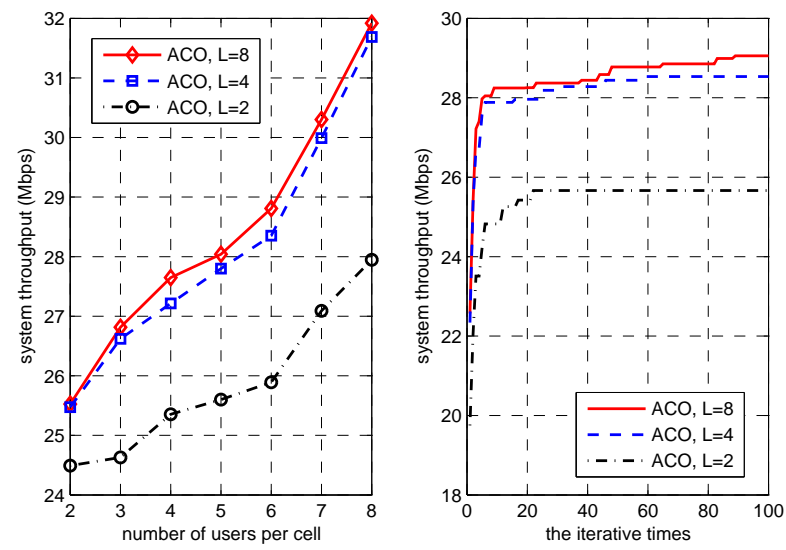

Fig. 4. (a) System throughput of different number of power levels; (b)Iterative trend of different number of power levels with $K=6$.

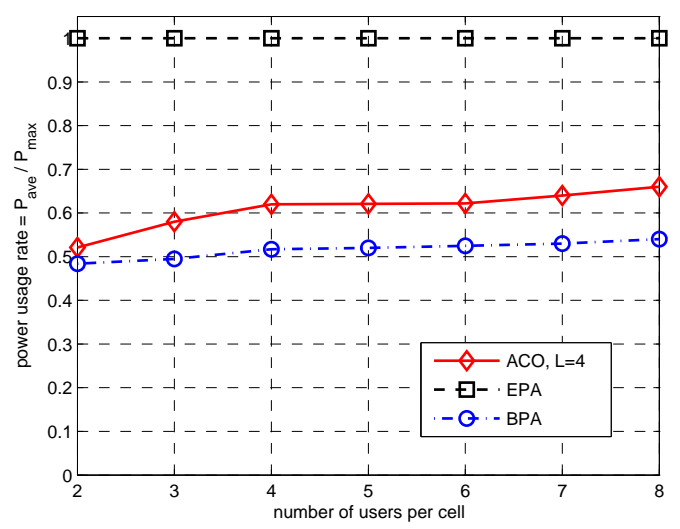

Fig. 5. Utilization of power of three different schemes with $L=4$.

improvement. Fig. 4 (b) further illustrates one time of searching progress of maximum system throughput with the iterative times for different power level number cases. It is shown that for all cases, the throughput improves significantly after 4-6 times of iteration, and after several times of searching, the simulation is close to convergence. Actually, we do not need wait for full convergence in practical, only few iterations to reach acceptable results is enough. $L=4$ is a suggested power level number, this is because it can reach good enough solution meanwhile the needed iterative time and computational dimensions is relatively low.

We also compare the utilization of power of different schemes. The ratio of average transmit power over BSs to the maximum power each BS can provide, which is denoted as power usage rate, is shown as a function of the number of users in Fig. 5. It can be seen clearly that EPA uses all the power provided. and BPA only consumes about half of the power constraint. Compared to BPA, ACO consumes more power, however the increased power usage rate is small, only about $10 \%$. It is worth to sacrifice this part of transmit power to gain the system throughput improvement.

\section{CONClusion}

The application of ACO to the power allocation problem in multi-cell OFDM systems with discrete power levels is studied in this paper. The objective is to maximize system throughput while subject to per-base-station power constraints. Simulation results show that the proposed algorithm can improve system throughput significantly compared with BPA and EPA, while the power usage rate is acceptable. Moreover, simulation results also indicate that with only 4 power levels, the proposed algorithm is able to achieve good enough result. In the further work, we'd like to focus on joint subchannel and power allocation problem in multi-cell scenarios, as well as further enhancing performance and reducing computational complexity as the dimension of the problem increases.

\section{ACKNOWLEDGMENT}

This work was supported by NSFC Project (No. 61001116, 61027003), International Scientific and Technological Cooperation Program (No.2010DFA11060). UK-China Science Bridges Project (No. EP/G042713/1)

\section{REFERENCES}

[1] 3GPP TR 25.892, "Feasibility Study for Orthogonal Frequency Division Multiplexing (OFDM) for UTRAN enhancement," June 2004.

[2] L. Venturino, N. Prasad, X. Wang, "An improved iterative water-filling algorithm for multi-cell interference mitigation in downlink OFDMA networks," in Proc. IEEE ACSSC'2007, vol. 41, pp. 1718-1722.

[3] H. Kwon, B. Gi Lee, "Distributed resource allocation through noncooperative game approach in multi-cell OFDMA systems," in Proc. IEEE ICC'2006, June 2006.

[4] H. Zhang, Q. Yang, F. Gao, Kyung Sup Kwak, "Distributed adaptive subchannel and power allocation for downlink OFDMA with inter-cell interference coordination," in Proc. IEEE GLOBECOM'2010, Dec. 2010.

[5] M. Andersin, Z. Rosberg, J. Zander,"Distributed discrete power control in cellular PCS", Wireless Personal Communications, 1998, vol. 6, No. 3, pp. 211-231.

[6] A. Gjendemsjø, D. Gesbert, G. Øien, S. Kiani, "Binary power control for sum rate maximization over multiple interfering links," IEEE Trans. on Wireless Commun., vol. 7, pp. 3164-3173, Aug. 2008.

[7] H. Zhang, L. Venturino, et al., "Weighted sum-Rate maximization in multi-cell networks via coordinated scheduling and discrete power control," Journal on Selected Areas in Communication, vol. 29, NO. 6, pp. 1214-1224, June 2011.

[8] Y. B. Reddy, V. V. Phoha, "Genetic algorithm approach for resource allocation in multi-user OFDM systems," in Proc. IEEE COMSWARE'2007.

[9] I. Ahmad, S. P. Majumder, "Adaptive resource allocation based on modified genetic algorithm and particle swarm optimization for multiuser OFDM systems," in Proc. IEEE ICECE, pp 211-216, Dec. 2008.

[10] H. Ahmadi, Y. H. Chew, "Subcarrier-and-bit allocation in multiclass multiuser single-cell OFDMA systems using an ant colony optimization based evolutionary algorithm," inProc. IEEE WCNC'2010, Apr. 2010.

[11] R. Lin, K. Niu, W. Xu, Z. He, "A two-level distributed sub-carrier allocation algorithm based on ant colony optimization in OFDMA systems," in Proc. IEEE VTC'2010-Spring, May 2010.

[12] A. J. Goldsmith, S.-G. Chua, "Variable-rate variable-power MQAM for fading channels," IEEE Trans. Commun., vol. 45, no. 10, pp. 1218-1230, Oct. 1997.

[13] Z. Luo, S. Zhang, "Dynamic spectrum management: Complexity and duality," IEEE J. Sel. Topics Signal Process., vol. 2, no. 1, Feb. 2008.

[14] A. Colorni, M. Dorigo, V. Maniezzo, "Distributed optimization by ant colonies," in Proc. ECAL91-Eur. Conf. Artificial Life. New York: Elsevier, pp. 134-142, 1991.

[15] M. Dorigo, L. M. Gambardella, "Ant colony system: A cooperative learning approach to the traveling salesman problem," IEEE Trans. Evol. Comput., vol. 1, pp. 53-66, Apr. 1997.

[16] M. Dorigo, T. Stüzle, "Ant colony optimization," MIT press, 305 pp, EISBN 0-262-04219-3 